\title{
Persistent Fetal Bradycardia
}

National Cancer Institute

\section{Source}

National Cancer Institute. Persistent Fetal Bradycardia. NCI Thesaurus. Code 692842.

A decrease in the fetal heart beat rate below 110 per minute or below the normal baseline value expected for the particular fetal age for a period of more than 10 minutes. 